\title{
A PRIORI ERROR ESTIMATE OF A DISCONTINUOUS FINITE VOLUME METHOD FOR THE OBSTACLE PROBLEM
}

\author{
YUPING ZENG*, TING LI \\ School of Mathematics, Jiaying University, Meizhou 514015, China \\ *Corresponding author: zeng_yuping@163.com \\ Received Jan. 2, 2021
}

\begin{abstract}
Aвstract. The present paper designs a discontinuous finite volume method for the numerical approximation of the obstacle problem. In addition, an optimal a priori error estimate in the energy norm is derived assuming some regularity conditions.

2010 Mathematics Subject Classification. 65N15; 65N30; $49 J 40$.

Key words and phrases. obstacle problem; variational inequality; discontinuous finite volume method; a priori error estimate.
\end{abstract}

\section{INTRODUCTION}

The obstacle problem describes the membrane deformation phenomenon that is an important example of a variational inequality of the first kind. The theory of variational inequalities has been made applications in diverse fields such as mechanics, physics, and operations research (cf. [27]). Due to the variational property, finite element (FE) method is a natural way to approximate the obstacle problem. In the last four decades, there has been made impressive progress in this field. More precisely, see $[7,10,14,22,23,40,45,56]$ for the conforming FE methods, and the nonconforming FE methods can be found in $[11,39,46]$. Recently, discontinuous Galerkin (DG) [1] methods have also been developed to solve the obstacle problem (cf. $[2,19,24,41,42,53])$.

Finite volume (FV) method is an efficient discrete scheme for solving partial differential equations (PDEs). By integrating the PDEs on a control volume, the FV method satisfies some conservation property such as mass, momentum, or energy. Thus, FV method become

DOI: 10.28924/APJM/8-17 
a popular approach in computational fluid mechanics. For more details of FV methods, we refer to the monographs $[21,32]$, the papers $[4,5,8,13,15-17,20,25,26,36,37,48-50,54,55]$ and references therein. Discontinuous finite volume (DFV) methods were originally introduced by Ye [51] for solving the second order elliptic equations, therein a priori error estimate in a meshdependent norm was derived. Inheriting attractive features of both DG and FV methods, such methods can easily handle complicate geometries and inhomogeneous boundary conditions, and they satisfy some conservative properties. Another interesting feature of DFV methods is their localizability of discontinuous elements and the corresponding dual partitions, this make them suitable for parallel computing. In addition, compared to the classical conforming and nonconforming FV methods, DFV methods have small support in the control volume of the dual mesh. For these reasons, DFV methods have further been investigated to solve various PDEs, such as second order elliptic equations $[3,12,28,35]$, Stokes equations $[9,18,30,44,52]$, Darcy-Stokes problems [34,43], Biot equations [29], phase field model [33], optimal control problems $[31,38]$ and so on.

In contrast to huge literature on FE methods for variational inequalities, the work on FV methods is considerably less developed. More recently, a conforming FV method is designed to solve two kinds of variational inequalities including the obstacle and simplified frictional problems [58]. Later, this method is extend to the Signorini problem and a super-close interpolation estimate was obtained (see [57]). The objective of this work is to apply the discontinuous finite volume (DFV) method to solve the obstacle problem and give an optimal error estimate in the energy norm. It is worth mentioning that we shall address two difficulties, one arises from the inherent nonlinearity of variational inequality, and the other comes from the complexity of bilinear form of DFV methods.

The rest of the paper is organized as follows. In Section 2, we introduce the model problem and state the corresponding DFV numerical scheme. Next, in Section 3 we give an optimal a priori error analysis in the mesh-dependent norm. Finally, some conclusions are made in Section 4.

\section{THE DFV METHOD FOR THE OBSTACLE PROBLEM}

2.1. Obstacle problem. We begin by introducing some notation. For $\mathcal{O} \in \mathbb{R}^{2}$, let $H^{k}(\mathcal{O}), k \geq 0$ be the usual Sobolev space with the norm $\|\cdot\|_{k, \mathcal{O}}$ and the seminorm $|\cdot|_{k, \mathcal{O}}$. When $\mathcal{O}=\Omega$, we omit the index $\Omega$. For $k=0, H^{0}(\mathcal{O})$ is reduced to the Lebesgue space $L^{2}(\mathcal{O})$. 
Let $\Omega \subset \mathbb{R}^{2}$ be a bounded convex polygonal domain with Lipschitz boundary $\Gamma$. For $f \in L^{2}(\Omega)$, we consider the elliptic obstacle problem: Find $u \in K$ such that

$$
\int_{\Omega} \nabla u \cdot \nabla(v-u) d x \geq \int_{\Omega} f(v-u) d x \quad \forall v \in K,
$$

with

$$
K=\left\{v \in H_{g}^{1}(\Omega): v \geq \psi \text { a.e. in } \Omega\right\} .
$$

Here $H_{g}^{1}(\Omega)=\left\{v \in H^{1}(\Omega): v=g\right.$ on $\left.\Gamma\right\}$, with $g$ being the restriction of an $H^{2}(\Omega)$ function to Г. $\psi \in H^{2}(\Omega)$ is referred to the obstacle function that satisfies $\psi \leq g$ on $\Gamma$.

2.2. DFV method. Let $\mathcal{T}_{h}$ ba a shape-regular mesh which decomposes $\Omega$ into triangular elements $\{T\}$. Denote by $h_{T}=\operatorname{diam}(T)$ and let $h=\max _{T \in \mathcal{T}_{h}} h_{T} \cdot \mathcal{E}_{h}^{I}$ stands for the set of interior edges of elements in $\mathcal{T}_{h}$, and $\mathcal{E}_{h}^{\partial}$ is the set of edges on the boundary $\Gamma$. Thus, the set of all edges $\mathcal{E}_{h}=\mathcal{E}_{h}^{I} \cup \mathcal{E}_{h}^{\partial}$. We assign each edge $e \in \mathcal{E}_{h}$ an unit normal $\mathbf{n}$, such that on $e \in \mathcal{E}_{h}^{\partial}$, $\mathbf{n}$ refers to the outward unit normal. In addition, we divide each element $T$ by connecting its vertices and barycenter to obtain a dual mesh $\mathcal{T}_{h}^{*}$, see Fig.1. In what follows, we shall use $C$, with or without subscripts to denote generic constants independent of $h$, but depend on the minimum angle of $T$.

For a discontinuous function $v$, on each interior edge $e \in \mathcal{E}_{h}^{I}$ shared by two elements $T^{+}$and $T^{-}$, let $v^{ \pm}=\left.v\right|_{e n \partial T^{ \pm}}$, we define the average and jump by

$$
\{v\}=\frac{1}{2}\left(v^{+}+v^{-}\right) \text {and } \llbracket v \rrbracket=v^{+}-v^{-} .
$$

On a boundary edge $e \in \mathcal{E}_{h}^{\partial}$, we define

$$
\{v\}=v \text { and } \llbracket v \rrbracket=v .
$$
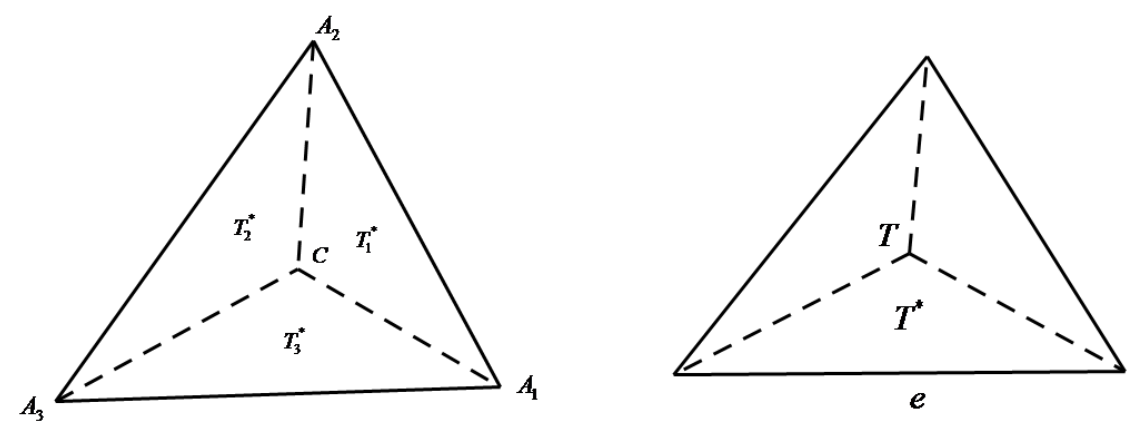

Figure 1. A triangular partition and its dual volume for DFV method. 
We consider the discontinuous $\mathbb{P}_{1}$ finite dimensional space for trial functions associated with $\mathcal{T}_{h}$ :

$$
V_{h}=\left\{v \in L^{2}(\Omega):\left.v\right|_{T} \in \mathbb{P}_{1}(T) \quad \forall T \in \mathcal{T}_{h}\right\}
$$

where $\mathbb{P}_{k}(T)$ is the space of polynomials of total degree at most $k$ on $T$. Then, an approximation of the set $K$ is defined by

$$
K_{h}=\left\{v_{h} \in V_{h}:\left.v_{h}\right|_{T}(p) \geq \psi(p) \quad \forall p \in \mathcal{V}_{T} \quad \forall T \in \mathcal{T}_{h}\right\}
$$

with $\mathcal{V}_{T}$ being the set of three vertices of $T$.

On the other hand, for test functions associated with dual mesh $\mathcal{T}_{h}^{*}$, we use the finite dimensional space:

$$
V_{h}^{*}=\left\{v \in L^{2}(\Omega):\left.v\right|_{T^{*}} \in \mathbb{P}_{0}\left(T^{*}\right) \quad \forall T^{*} \in \mathcal{T}_{h}^{*}\right\}
$$

Let $V(h)=V_{h}+\left[H_{g}^{1}(\Omega) \cap H^{2}(\Omega)\right]$, in order to connect $V(h)$ to $V_{h}^{*}$, we define the mapping $\gamma_{h}: V(h) \rightarrow V_{h}^{*}$ by

$$
\left.\gamma_{h} v\right|_{T^{*}}=\left.\frac{1}{h_{e}} \int_{e} v\right|_{T^{*}} d s \quad \forall T^{*} \in \mathcal{T}_{h}^{*},
$$

where $h_{e}$ is the length of the edge $e$.

Following [51], we define the bilinear form with regard to the DFV method:

$$
\begin{aligned}
a_{h}(w, v)= & A(w, v)-\sum_{e \in \mathcal{E}_{h}} \int_{e}\{\nabla w \cdot \mathbf{n}\} \llbracket \gamma_{h} v \rrbracket d s-\sum_{e \in \mathcal{E}_{h}} \int_{e}\{\nabla v \cdot \mathbf{n}\} \llbracket \gamma_{h} w \rrbracket d s \\
& +\sum_{e \in \mathcal{E}_{h}} \rho_{e} h_{e}^{-1} \int_{e} \llbracket \gamma_{h} w \rrbracket \llbracket \gamma_{h} v \rrbracket d s
\end{aligned}
$$

with $A(w, v)=-\sum_{T^{*} \in \mathcal{T}_{h}^{*}} \int_{\partial T^{*}}(\nabla w \cdot \mathbf{n}) \gamma_{h} v d s+\sum_{T \in \mathcal{T}_{h}} \int_{\partial T}(\nabla w \cdot \mathbf{n}) \gamma_{h} v d s$.

The DFVM for solving the obstacle problem (1) is to find $u_{h} \in K_{h}$ such that

$$
\begin{aligned}
& a_{h}\left(u_{h}, v_{h}-u_{h}\right) \geq \int_{\Omega} f\left(\gamma_{h} v_{h}-\gamma_{h} u_{h}\right) d x-\sum_{e \in \mathcal{E}_{h}^{\partial}} \int_{e} \gamma_{h} g\left(\nabla\left(v_{h}-u_{h}\right) \cdot \mathbf{n}\right) d s \\
& \quad+\sum_{e \in \mathcal{E}_{h}^{\partial}} \rho_{e} h_{e}^{-1} \int_{e} \gamma_{h} g\left(\gamma_{h} v_{h}-\gamma_{h} u_{h}\right) d s \quad \forall v_{h} \in K_{h} .
\end{aligned}
$$

\section{A PRIORI ERROR ESTIMATES}

This subsection is devoted to a priori error estimate of the numerical scheme (3). To proceed, we first define the mesh-dependent norm $\|\cdot\|_{h}$ on $V(h)$ (cf. [51]):

$$
\|v\|_{h}=\left(\sum_{T \in \mathcal{T}_{h}}\|\nabla v\|_{0, T}^{2}+\sum_{e \in \mathcal{E}_{h}} \llbracket \gamma_{h} v \rrbracket^{2}+\sum_{T \in \mathcal{T}_{h}} h_{T}^{2}|v|_{2, T}^{2}\right)^{1 / 2} .
$$


It can be proved that $a_{h}(\cdot, \cdot)$ satisfies the following properties (see Lemmas 2.2 and 2.3 in [51] for more details).

Lemma 3.1. There holds

$$
\left|a_{h}(w, v)\right| \leq C_{\alpha}\|w\|_{h}\|v\|_{h} \quad \forall w, v \in V(h) .
$$

Furthermore, we can choose large enough $\rho_{e}$ to satisfy

$$
a_{h}(v, v) \geq C_{\beta}\|v\|_{h}^{2} \quad \forall v \in V_{h}
$$

We also need some useful results with respect to the mapping $\gamma_{h}$ (cf. [17]).

\section{Lemma 3.2.}

$$
\begin{gathered}
\text { if } \llbracket v \rrbracket=0, \text { then } \quad \llbracket \gamma_{h} v \rrbracket=0, \\
\left\|v-\gamma_{h} v\right\|_{0, T} \leq C h_{T}\|\nabla v\|_{0, T} \quad \forall v \in V(h),
\end{gathered}
$$

Let $u_{I}$ be the the continuous linear interpolation of $u$, it satisfies $[6,47]$ :

$$
\left\|u-u_{I}\right\|_{m, T} \leq C h^{2-m}|u|_{2, T} \quad m=0,1,2 .
$$

In addition, we recall the following trace inequality (see e.g. [1]):

$$
\|w\|_{0, e}^{2} \leq C\left(h_{e}^{-1}\|w\|_{0, T}^{2}+h_{e}\|\nabla w\|_{0, T}^{2}\right) \quad \forall w \in H^{1}(T)
$$

with $e$ any edge of the element $T$.

Observing that $\llbracket u \rrbracket=0$ and $\llbracket u_{I} \rrbracket=0$ on any $e \in \mathcal{E}_{h}^{I}$, from (7) we see that $\llbracket \gamma_{h} u \rrbracket=0$ and $\llbracket \gamma_{h} u_{I} \rrbracket=0$ on any $e \in \mathcal{E}_{h}^{I}$. Therefore,

$$
\left\|u-u_{I}\right\|_{h}=\left(\sum_{T \in \mathcal{T}_{h}}\left(\left\|\nabla\left(u-u_{I}\right)\right\|_{0, T}^{2}+h_{T}^{2}\left|u-u_{I}\right|_{2, T}^{2}\right)+\sum_{e \in \mathcal{E}_{h}^{\partial}} \llbracket \gamma_{h}\left(u-u_{I}\right) \rrbracket^{2}\right)^{1 / 2}
$$


In view of the definition of $\gamma_{h}$, Cauchy-Schwarz inequality and trace inequality (10), we deduce that

$$
\begin{aligned}
\sum_{e \in \mathcal{E}_{h}^{\partial}} \llbracket \gamma_{h}\left(u-u_{I}\right) \rrbracket^{2} & =\sum_{e \in \mathcal{E}_{h}^{\partial}} h_{e}^{-2}\left(\int_{e}\left(u-u_{I}\right) d s\right)^{2} \\
& \leq \sum_{e \in \mathcal{E}_{h}^{\partial}} h_{e}^{-2} \int_{e} 1^{2} d s \int_{e}\left(u-u_{I}\right)^{2} d s \\
& =\sum_{e \in \mathcal{E}_{h}^{\partial}} h_{e}^{-1}\left\|u-u_{I}\right\|_{0, e}^{2} \\
& \leq C \sum_{T \in \mathcal{T}_{h}}\left(h_{e}^{-2}\left\|u-u_{I}\right\|_{0, T}^{2}+\left\|\nabla\left(u-u_{I}\right)\right\|_{0, T}^{2}\right) .
\end{aligned}
$$

Inserting (12) into (11), and using (9) and the shape-regularity of the mesh, we infer that

$$
\left\|u-u_{I}\right\|_{h} \leq C h|u|_{2}
$$

We are now in a position to state our main result. Applying some techniques developed in $[7,53]$, we prove that the DFV method has an optimal convergence rate in the meshdependent norm.

Theorem 3.3. Let $u$ and $u_{h}$ be the solutions of (1) and (3), respectively. Assume that $u \in H^{2}(\Omega)$, it holds that

$$
\left\|u-u_{h}\right\|_{h} \leq C h\left(|u|_{2}+|\psi|_{2}+\|\Delta u+f\|_{0}\right)
$$

Proof. The triangle inequality yields

$$
\left\|u-u_{h}\right\|_{h} \leq\left\|u-u_{I}\right\|_{h}+\left\|u_{I}-u_{h}\right\|_{h} .
$$

The bound of $\left\|u-u_{I}\right\|_{h}$ have been obtained in (13), we only need to estimate $\left\|u_{I}-u_{h}\right\|_{h}$. It follows from (6) that

$$
C_{\beta}\left\|u_{I}-u_{h}\right\|_{h}^{2} \leq a_{h}\left(u_{I}-u_{h}, u_{I}-u_{h}\right) \equiv \mathbb{B}_{1}+\mathbb{B}_{2}
$$

where

$$
\begin{aligned}
& \mathbb{B}_{1}=a_{h}\left(u_{I}-u, u_{I}-u_{h}\right), \\
& \mathbb{B}_{2}=a_{h}\left(u-u_{h}, u_{I}-u_{h}\right) .
\end{aligned}
$$

For the first term $\mathbb{B}_{1}$, application of (5) and Young's inequality to find that

$$
\mathbb{B}_{1} \leq C_{\alpha}\left\|u_{I}-u\right\|_{h}\left\|u_{I}-u_{h}\right\|_{h} \leq \frac{C_{\alpha}}{4 \epsilon_{1}}\left\|u_{I}-u\right\|_{h}^{2}+C_{\alpha} \epsilon_{1}\left\|u_{I}-u_{h}\right\|_{h}^{2}
$$


It remains to estimate the second term $\mathbb{B}_{2}$. Noting that $\llbracket u \rrbracket=0\left(\forall e \in \mathcal{E}_{h}^{I}\right)$, this together with (7) gives $\llbracket \gamma_{h} u \rrbracket=0\left(\forall e \in \mathcal{E}_{h}^{I}\right)$. In addition, since $V_{h}^{*}$ is piecewise constant space and $\llbracket \nabla u \cdot \mathbf{n} \rrbracket=0\left(\forall e \in \mathcal{E}_{h}^{I}\right)$, we use integration by parts to obtain

$$
\begin{aligned}
& a_{h}\left(u, u_{I}-u_{h}\right) \\
& =-\sum_{T^{*} \in \mathcal{T}_{h}^{*}} \int_{\partial T^{*}}(\nabla u \cdot \mathbf{n}) \gamma_{h}\left(u_{I}-u_{h}\right) d s+\sum_{T \in \mathcal{T}_{h}} \int_{\partial T}(\nabla u \cdot \mathbf{n}) \gamma_{h}\left(u_{I}-u_{h}\right) d s \\
& -\sum_{e \in \mathcal{E}_{h}} \int_{e}\{\nabla u \cdot \mathbf{n}\} \llbracket \gamma_{h}\left(u_{I}-u_{h}\right) \rrbracket d s-\sum_{e \in \mathcal{E}_{h}^{\partial}} \int_{e} \gamma_{h} g \nabla\left(u_{I}-u_{h}\right) d s \\
& +\sum_{e \in \mathcal{E}_{h}^{\partial}} \rho_{e} h_{e}^{-1} \int_{e} \gamma_{h} g \gamma_{h}\left(u_{I}-u_{h}\right) d s \\
& =\sum_{T^{*} \in \mathcal{T}_{h}^{*}} \int_{T^{*}}-\Delta u \gamma_{h}\left(u_{I}-u_{h}\right) d x+\sum_{e \in \mathcal{E}_{h}} \int_{e}\{\nabla u \cdot \mathbf{n}\} \llbracket \gamma_{h}\left(u_{I}-u_{h}\right) \rrbracket d s \\
& +\sum_{e \in \mathcal{E}_{h}^{I}} \int_{e} \llbracket \nabla u \cdot \mathbf{n} \rrbracket\left\{\gamma_{h}\left(u_{I}-u_{h}\right)\right\} d s-\sum_{e \in \mathcal{E}_{h}} \int_{e}\{\nabla u \cdot \mathbf{n}\} \llbracket \gamma_{h}\left(u_{I}-u_{h}\right) \rrbracket d s \\
& -\sum_{e \in \mathcal{E}_{h}^{\partial}} \int_{e} \gamma_{h} g \nabla\left(u_{I}-u_{h}\right) d s+\sum_{e \in \mathcal{E}_{h}^{\partial}} \rho_{e} h_{e}^{-1} \int_{e} \gamma_{h} g \gamma_{h}\left(u_{I}-u_{h}\right) d s \\
& =\sum_{T \in \mathcal{T}_{h}} \int_{T}-\Delta u \gamma_{h}\left(u_{I}-u_{h}\right) d x-\sum_{e \in \mathcal{E}_{h}^{\partial}} \int_{e} \gamma_{h} g \nabla\left(u_{I}-u_{h}\right) d s \\
& +\sum_{e \in \mathcal{E}_{h}^{\partial}} \rho_{e} h_{e}^{-1} \int_{e} \gamma_{h} g \gamma_{h}\left(u_{I}-u_{h}\right) d s .
\end{aligned}
$$

On the other hand, setting $v_{h}=u_{I}$ in (3) shows that

$$
\begin{aligned}
a_{h}\left(u_{h}, u_{I}-u_{h}\right) \geq & \int_{\Omega} f \gamma_{h}\left(u_{I}-u_{h}\right) d x-\sum_{e \in \mathcal{E}_{h}^{\partial}} \int_{e} \gamma_{h} g \nabla\left(u_{I}-u_{h}\right) d s \\
& +\sum_{e \in \mathcal{E}_{h}^{\partial}} \rho_{e} h_{e}^{-1} \int_{e} \gamma_{h} g \gamma_{h}\left(u_{I}-u_{h}\right) d s .
\end{aligned}
$$

Then, we infer from (18) and (19) that

$$
\begin{aligned}
\mathbb{B}_{2} & =a_{h}\left(u-u_{h}, u_{I}-u_{h}\right) \\
& \leq \sum_{T \in \mathcal{T}_{h}} \int_{T}-(\Delta u+f) \gamma_{h}\left(u_{I}-u_{h}\right) d x
\end{aligned}
$$




$$
\begin{aligned}
&= {\left[\sum_{T \in \mathcal{T}_{h}} \int_{T}-(\Delta u+f) \gamma_{h}\left(u_{I}-u_{h}\right) d x-\sum_{T \in \mathcal{T}_{h}} \int_{T}-(\Delta u+f)\left(u_{I}-u_{h}\right) d x\right] } \\
&+\sum_{T \in \mathcal{T}_{h}} \int_{T}-(\Delta u+f)\left(u_{I}-u_{h}\right) d x \\
& \equiv \mathbb{B}_{21}+\mathbb{B}_{22} .
\end{aligned}
$$

For simplicity, let $\vartheta=u_{I}-u_{h}, \mathbb{B}_{21}$ can be rewritten as

$$
\mathbb{B}_{21}=\sum_{T \in \mathcal{T}_{h}} \int_{T}-(\Delta u+f)\left(\gamma_{h} \vartheta-\vartheta\right) d s
$$

In term of (8), Cauchy-Schwarz inequality and Young's inequality, we can estimate $\mathbb{B}_{21}$ by

$$
\begin{aligned}
\mathbb{B}_{21} & =\sum_{T \in \mathcal{T}_{h}} \int_{T}-(\Delta u+f)\left(\gamma_{h} \vartheta-\vartheta\right) d s \\
& \leq\left(\sum_{T \in \mathcal{T}_{h}}\|\Delta u+f\|_{0, T}^{2}\right)^{1 / 2}\left(\sum_{T \in \mathcal{T}_{h}}\left\|\gamma_{h} \vartheta-\vartheta\right\|_{0, T}^{2}\right)^{1 / 2} \\
& \leq\left(\sum_{T \in \mathcal{T}_{h}}\|\Delta u+f\|_{0, T}^{2}\right)^{1 / 2}\left(\sum_{T \in \mathcal{T}_{h}} C_{1} h_{T}^{2}|\vartheta|_{1, T}^{2}\right)^{1 / 2} \\
& \leq C_{2} h\|\Delta u+f\|_{0}\left\|u_{I}-u_{h}\right\|_{h} \\
& \leq \frac{C_{2}}{4 \epsilon_{2}} h^{2}\|\Delta u+f\|_{0}+C_{2} \epsilon_{2}\left\|u_{I}-u_{h}\right\|_{h}^{2} .
\end{aligned}
$$

Next, we shall give the bound for $\mathbb{B}_{22}$. First, we recall the following well-known result [7]

$$
-\Delta u \geq f, u \geq \psi,(-\Delta u-f)(u-\psi)=0 \text { a.e. in } \Omega .
$$

By adding and subtracting some terms, we have

$$
\begin{aligned}
\mathbb{B}_{22}= & \sum_{T \in \mathcal{T}_{h}} \int_{T}-(\Delta u+f)\left(u_{I}-u_{h}\right) d x \\
= & \sum_{T \in \mathcal{T}_{h}} \int_{T}-(\Delta u+f)\left(u_{I}-u\right) d x+\sum_{T \in \mathcal{T}_{h}} \int_{T}-(\Delta u+f)(u-\psi) d x \\
& +\sum_{T \in \mathcal{T}_{h}} \int_{T}-(\Delta u+f)\left(\psi_{I}-u_{h}\right) d x+\sum_{T \in \mathcal{T}_{h}} \int_{T}-(\Delta u+f)\left(\psi-\psi_{I}\right) d x .
\end{aligned}
$$

Due to (23), the second term in the right hand of (24) satisfies

$$
\sum_{T \in \mathcal{T}_{h}} \int_{T}-(\Delta u+f)(u-\psi) d x=0 .
$$


On the other hand, by the definition of $K_{h}$, it holds that $\left.u_{h}\right|_{T}(p) \geq \psi(p)=\psi_{I}(p),(\forall p \in$ $\left.\mathcal{V}_{T}, \forall T \in \mathcal{T}_{h}\right)$. Since we use linear finite element, it follows that $\psi_{I}-u_{h} \leq 0$ on any $T \in \mathcal{T}_{h}$.

Consequently, we have

$$
\sum_{T \in \mathcal{T}_{h}} \int_{T}-(\Delta u+f)\left(\psi_{I}-u_{h}\right) d x \leq 0 .
$$

Inserting (25) and (26) into (24) yields

$$
\mathbb{B}_{22} \leq \sum_{T \in \mathcal{T}_{h}} \int_{T}-(\Delta u+f)\left(u_{I}-u\right) d x+\sum_{T \in \mathcal{T}_{h}} \int_{T}-(\Delta u+f)\left(\psi-\psi_{I}\right) d x .
$$

Utilizing the interpolation error estimates (9) and Cauchy-Schwarz inequality implies that

$$
\mathbb{B}_{22} \leq C_{3} h^{2}\|\Delta u+f\|_{0}\left(|u|_{2}+|\psi|_{2}\right)
$$

Combining (16), (17), (20), (22) and (28), we infer that

$$
\begin{aligned}
& \left(C_{\beta}-C_{\alpha} \epsilon_{1}-C_{2} \epsilon_{2}\right)\left\|u_{I}-u_{h}\right\|_{h}^{2} \\
& \leq \frac{C_{\alpha}}{4 \epsilon_{1}}\left\|u_{I}-u\right\|_{h}^{2}+\frac{C_{2}}{4 \epsilon_{2}} h^{2}\|\Delta u+f\|_{0}+C_{3} h^{2}\|\Delta u+f\|_{0}\left(|u|_{2}+|\psi|_{2}\right) .
\end{aligned}
$$

Choosing appropriate parameters $\epsilon_{i}(i=1,2)$ such that $C_{\beta}-C_{\alpha} \epsilon_{1}-C_{2} \epsilon_{2}>0$, and combining (13), (15) and (29), we obtain the desired estimate (14).

\section{Conclusion}

We introduced and analyzed a discontinuous finite volume method to solve the obstacle problem. A detailed a priori error estimate in the energy norm was established. Nature extension of this work includes a posteriori error estimate, and we shall also extend such method to variational inequality of the second kind in the future work.

\section{AcKNOWledgments}

This work was supported by Guangdong Basic and Applied Basic Research Foundation (Grant No. 2020A1515011032).

\section{REFERENCES}

[1] D. N. Arnold, F. Brezzi, B. Cockburn and L. D. Marini, Unified analysis of discontinuous Galerkin methods for elliptic problems, SIAM J. Numer. Anal. 39 (2002), 1749-1779.

[2] L. Banz and E. P. Stephan, A posteriori error estimates of $h p$-adaptive IPDG-FEM for elliptic obstacle problems, Appl. Numer. Math. 76 (2014), 76-92. 
[3] C. Bi and J. Geng, A discontinuous finite volume element method for second-order elliptic problems, Numer. Methods PDEs. 28 (2012), 425-440.

[4] C. Bi, Y. Lin and M. Yang, Finite volume element method for monotone nonlinear elliptic problems, Numer. Methods PDEs. 29 (2013), 1097-1120.

[5] C. Bi and H. Rui, Uniform convergence of finite volume element method with Crouzeix-Raviart element for non-self-adjoint and indefinite elliptic problems, J. Comput. Appl. Math. 200 (2007), 555-565.

[6] S. C. Brenner and L. R. Scott, The Mathematical Theory of Finite Element Methods (3rd edn), Springer-Verlag, New York, 2008.

[7] F. Brezzi, W. W. Hager and P. A. Raviart, Error estimates for the finite element solution of variational inequalities, Part I: primal theory, Numer. Math. 28 (1977), 431-443.

[8] Z. Cai, J. Mandel and S. McCormick, The finite volume element method for diffusion equations on general triangulations, SIAM J. Numer. Anal. 28 (1991), 392-402.

[9] C. Carstensen, K. Dond, N. Nataraj and A. K. Pani, Three First-order finite volume element methods for Stokes equations under minimal regularity assumptions, SIAM J. Numer. Anal. 56 (2018), 2648-2671.

[10] C. Carstensen and J. Hu, An optimal adaptive finite element method for an obstacle problem, Comput. Methods Appl. Math. 15 (2015), 259-277.

[11] C. Carstensen and K. Köhler, Nonconforming FEM for the obstacle problem, IMA J. Numer. Anal. 37 (2017), 64-93.

[12] C. Carstensen, N. Nataraj and A. K. Pani, Comparison results and unified analysis for first-order finite volume element methods for a Poisson model problem, IMA. J. Numer. Anal. 36 (2016), 1120-1142.

[13] L. Chen, A new class of high order finite volume methods for second order elliptic equations, SIAM J. Numer. Anal. 47 (2010), 4021-4043.

[14] Z. Chen and R. Nochetto, Residual type a posteriori error estimates for elliptic obstacle problems, Numer. Math. 84 (2000), 527-548.

[15] Z. Chen, Y. Xu and Y. Zhang, A construction of higher-order finite volume methods, Math. Comput. 84 (2015), 599-628.

[16] S. H. Chou and Q. Li, Error estimates in $L^{2}, H^{1}$, and $L^{\infty}$ in covolume methods for elliptic and parabolic problems, a unified approach, Math. Comput. 69 (2000), 103-120.

[17] S. H. Chou and X. Ye, Unified analysis of finite volume methods for second order elliptic problems, SIAM J. Numer. Anal. 45 (2007), 1639-1653.

[18] M. Cui and X. Ye, Unified analysis of finite volume methods for the Stokes equations, SIAM J. Numer. Anal. 48 (2010), 824-839.

[19] J. K. Djoko, Discontinuous Galerkin finite element methods for variational inequalities of first and second kinds, Numer. Methods PDEs. 24 (2008), 296-311.

[20] R. E. Ewing, T. Lin and Y. Lin, On the accuracy of the finite volume element method based on piecewise linear polynomials, SIAM J. Numer. Anal. 39 (2002), 1865-1888.

[21] R. Eymard, T. Gallouët and R. Herbin, Finite Volume Methods: Handbook of Numerical Analysis, NorthHolland, Amsterdam, 2000. 
[22] R. S. Falk, Error estimates for the approximation of a class of variational inequalities, Math. Comput. 28 (1974), 963-971.

[23] R. Glowinski, Numerical Methods for Nonlinear Variational Problems, Springer-Verlag, Berlin, 2008.

[24] T. Gudi and P. Kamana, A posteriori error control of discontinuous Galerkin methods for elliptic obstacle problems, Math. Comput. 83 (2014), 579-602.

[25] L. Guo, H. Li and Q. Zou, Interior estimate of finite volume element methods over quadrilateral meshes for elliptic equations, SIAM J. Numer. Anal. 57 (2019), 2246-2265.

[26] J. Huang and S. Xi, On the finite volume element method for general self-adjoint elliptic problems, SIAM Numer. Anal. 35 (1998), 1762-1774.

[27] D. Kinderlehrer and G. Stampacchia, An Introduction to Variational Inequalities and Their Applications, Academic Press, New York, 1980.

[28] S. Kumar, N. Nataraj and A. K. Pani, Discontinuous Galerkin finite volume element methods for second-order linear elliptic problems, Numer. Methods PDEs. 25 (2009), 1402-1424.

[29] S. Kumar, R. Oyarzúa, R. Ruiz-Baier and R. Sandilya, Conservative discontinuous finite volume and mixed schemes for a new four-field formulation in poroelasticity, ESAIM: M2AN. 54 (2020), 273-299.

[30] S. Kumar and R. Ruiz-Baier, Equal order discontinuous finite volume element methods for the Stokes problem, J. Sci Comput. 65 (2015), 956-978.

[31] S. Kumar R. Ruiz-Baier and R. Sandilya, Error bounds for discontinuous finite volume discretisations of Brinkman optimal control problems, J. Sci. Comput. 78 (2019), 64-93.

[32] R. Li, Z. Chen and W. Wu, Generalized Difference Methods for Differential Equations: Numerical Analysis of Finite Volume Methods, Marcel Dekker, New York, 2000.

[33] R. Li, Y. Gao, J. Chen, L. Zhang, X. He and Z. Chen, Discontinuous finite volume element methodfor a coupled Navier-Stokes-Cahn-Hilliard phase field model, Adv. Comput. Math. 46 (2020), 1-35.

[34] R. Li, Y. Gao, J. Li and Z. Chen, Discontinuous finite volume element method for a coupled nonstationary Stokes-Darcy problem, J. Sci. Comput. 74 (2018), 693-727.

[35] J. Liu, L. Mu, X. Ye and R. Jari, Convergence of the discontinuous finite volume method for elliptic problems with minimal regularity, J. Comput. Appl. Math. 236 (2012), 4537-4546.

[36] X. Luo, Y. Chen, Y. Huang and T. Hou, Some error estimates of finite volume element approximation for parabolic optimal control problems, Optim. Control Appl. Meth. 35 (2014), 145-165.

[37] J. Lv and Y. Li, Optimal biquadratic finite volume element methods on quadrilateral meshes, SIAM J. Numer. Anal. 50 (2012), 2379-2399.

[38] R. Sandilya and S. Kumar, Convergence analysis of discontinuous finite volume methods for elliptic optimal control problems, Int. J. Comput. Methods. 13 (2015), 1640012

[39] D. Shi, C. Wang and Q. Tang, Anisotropic crouzeix-raviart type nonconforming finite element methods to variational inequality problem with displacement obstacle, J. Comput. Math. 33 (2015) 86-99.

[40] A. Veeser, Efficient and reliable a posteriori error estimators for elliptic obstacle problems, SIAM J. Numer. Anal. 39 (2001), 146-167. 
[41] F. Wang, W. Han and X. Cheng, Discontinuous Galerkin methods for solving elliptic variational inequalities, SIAM J. Numer. Anal. 48 (2010), 708-733.

[42] F. Wang, W. Han, J. Eichholz and X. Chen, A posteriori error estimates for discontinuous Galerkin methods of obstacle problems, Nonlinear Anal. RWA. 22 (2015), 664-679.

[43] G. Wang, Y. He and R. Li, Discontinuous finite volume methods for the stationary Stokes-Darcy problem, Int. J. Numer. Methods Engrg. 107 (2016), 395-418.

[44] J. Wang, Y. Wang, X. Ye, A unified a posteriori error estimator for finite volume methods for the Stokes equations, Math. Methods Appl. Sci. 41 (2018), 866-880.

[45] L. Wang, On the quadratic finite element approximation to the obstacle problem, Numer. Math. 92 (2002), 771-778.

[46] L. Wang, On the error estimate of nonconforming finite element approximation to the obstacle problem, J. Comput. Math. 21 (2003), 481-490.

[47] M. Wang and Z. Shi, Finite Element Methods, Beijing: Science Press, 2010.

[48] H. Wu and R. Li, Error estimates for finite volume element methods for general second-order elliptic problems, Numer. Methods PDEs. 19 (2003), 693-708.

[49] J. Xu and Q. Zou, Analysis of linear and quadratic simplicial finite volume methods for elliptic equations, Numer. Math. 111 (2009), 469-492.

[50] M. Yang and J. Liu, A quadratic finite volume element method for parabolic problems on quadrilateral meshes, IMA J. Numer. Anal. 31 (2011), 1038-1061.

[51] X. Ye, A new discontinuous finite volume method for elliptic problems, SIAM J. Numer. Anal. 42 (2004), 1062-1072.

[52] X. Ye, A discontinuous finite volume method for the Stokes problems, SIAM J. Numer. Anal. 44 (2006), 183-198.

[53] Y. Zeng, J. Chen and F. Wang, Error estimates of the weakly over-penalized symmetric interior penalty method for two variational inequalities, Comput. Math. Appl. 69 (2015), 760-770.

[54] Y. Zhang, M. Yang and C. Chen, The hybrid Wilson finite volume method for elliptic problems on quadrilateral meshes, Adv. Comput. Math. 45 (2019), 429-452.

[55] Z. Zhang and Q. Zou, Vertex centered finite volume schemes of any order over quadrilateral meshes for elliptic boundary problems, Numer. Math. 130 (2015), 363-393.

[56] Q. Zou, A. Veeser, R. Kornhuber and C. Cräser, Hierarchical error estimates for the energy functional in obstacle problems, Numer. Math. 117 (2011), 653-677.

[57] T. Zhang and Z. Li, An analysis of finite volume element method for solving the Signorini problem, Appl. Math. Comput. 270 (2015), 830-841.

[58] T. Zhang and L. Tang, Finite volume method for the variational inequalities of first and second kinds, Math. Methods Appl. Sci. 38 (2015), 3980-3989. 